\title{
Flavanones inhibit the clonogenicity of HCT116 cololectal cancer cells
}

\author{
YOONKYUNG WOO $^{1 *}$, SOON YOUNG SHIN ${ }^{2 *}$, JIYE HYUN $^{1}$, SUNG DAE LEE $^{3}$, \\ YOUNG HAN LEE ${ }^{2}$ and YOONGHO LIM ${ }^{1}$
}

\author{
${ }^{1}$ Division of Bioscience and Biotechnology, BMIC, Konkuk University, MoleNBio, Seoul 143-701; \\ ${ }^{2}$ Department of Biomedical Science and Technology, SMART-Institute of Advanced Biomedical Science, \\ RCTC, Konkuk University, Seoul 143-701; ${ }^{3}$ Swine Science Division, National Institute of \\ Animal Science, RDA, Cheonan 330-801, Republic of Korea
}

Received October 18, 2011; Accepted December 1, 2011

DOI: $10.3892 / \mathrm{ijmm} .2011 .857$

\begin{abstract}
Naringenin has been shown to display various biological effects such as antioxidant, anticancer, anti-inflammatory, and antiviral activities. Taxifolin inhibits the production of lipopolysaccharide-induced prostaglandin E, and fustin suppresses the activity of acetylcholinesterase. They all belong to flavanone which is a class of flavonoids with a C6-C3-C6 skeleton. Since the anticancer activities of flavanone derivatives have rarely been reported, we examined the effects of 26 flavanone derivatives on HCT116 colorectal cancer cells. Our results suggest that flavanone derivatives control the expression of cell cycle regulatory proteins, which blocks G1 cell cycle progression and inhibits the clonogenicity of HCT116 cells. In addition, in order to design flavanone derivatives that show better anticancer activity, structure-activity relationships were examined.
\end{abstract}

\section{Introduction}

A trihydroxyflavanone, naringenin, shows various biological effects such as antioxidant, anticancer, anti-inflammatory, and antiviral activities (1). Taxifolin, a pentahydroxyflavanone, inhibits the production of lipopolysaccharide-induced prostaglandin $\mathrm{E}$ and the expression of inflammatory mediators, and shows analgesic activity (2-4). Fustin, a tetrahydroxyflavanone, reduces $\beta$-amyloid-induced learning impairment,

Correspondence to: Professor Yoongho Lim, Division of Bioscience and Biotechnology, Konkuk University, Hwayang-Dong 1, Kwangjin-Gu, Seoul 143-701, Republic of Korea

E-mail: yoongho@konkuk.ac.kr

Professor Young Han Lee, Department of Biomedical Science and Technology, Konkuk University, Hwayang-Dong 1, Kwangjin-Gu, Seoul 143-701, Republic of Korea

E-mail: yhlee58@konkuk.ac.kr

${ }^{*}$ Contributed equally

Key words: flavanone, colorectal cancer, HCT116 cell, quantitatively SAR, clonogenicity the activity of acetylcholinesterase, and the gene expression induced by $\beta$-amyloid, and shows a dose-independent antimutagenic activity (5-7). Pinocembrin, a dihydroxyflavanone, improves neurological deficits and the survival of Epilachna paenulata, soothes hippocampal neuronal loss, and increases the activity of heme oxygenase (8-10). Alpinetin, 7-hydroxy5-methoxyflavanone, has demonstrated cytotoxic activity against human cancer cells such as MCF7 and Caski cells (11). They all belong to flavanones which are a class of flavonoids with a C6-C3-C6 skeleton. Here, the C6-C3 of chroman-4-one and $\mathrm{C} 6$ of phenyl ring were connected to each other through a carbon-carbon single bond. Because chroman-4-one contains a chiral carbon, the structure of flavanone is flexible. The wide range of the flavanone bioactivity may be due to its chiral structure $(12,13)$. Studies on flavanones have recently been carried out. More than 1,000 research articles on flavonones can be found on PubMed, with about $20 \%$ of them published within the last 3 years. Lipinski's rule of 5 is a rule of thumb to evaluate drug-like compounds, and revelas that flavanones belong to the category (14).

Colorectal cancer is the third most common cancer. More than 100,000 new patients were diagnosed with this cancer in the United States in 2010 and more than 50,000 died from this disease (15). Even though the factors causing colorectal cancer are not clear, Western style diet, and consumption of red meat or alcohol increase the person's risk. The lifetime risk of colorectal cancer is about 7\% (16). Many drugs including 5-fluorouracil are known for their chemotherapeutic properties. Most chemotherapeutic agents show cellular toxicities which affect cancer as well as normal cells (17). Therefore, the development of new drugs showing low toxicities is required. More than 10,000 flavonoids have been reported, but they are not used as chemotherapeutic agents because of their mild activities. Studies on adjuvant colorectal cancer therapy have recently been performed (16). Flavonoids do not show sufficient activity to be used for chemotherapy, but they can be applied for adjuvant therapy. In addition, the anticancer activities of flavanones have rarely been reported. We examined the effects of 26 flavanone derivatives on HCT116 colorectal cancer cells. To examine their anticancer effects, the clonogenicity of HCT116 human colorectal carcinoma cells as a long-term 
survival assay was measured. The aim of this research was to find the structural conditions of flavanones that would result in optimal anticancer activity against colorectal cancer cells based on a comparison of structures of the flavanone derivatives and their inhibitory effects against the clonogenicity.

\section{Materials and methods}

Flavanone derivatives. Five monohydroxyflavanones, a trihydroxyflavanone, a tetrahydroxyflavanone, a pentahydroxyflavanone, 4 monomethoxyflavanones, 4 dimethoxyflavanones, 5 trimethoxyflavanones, 4 hydroxymethoxyflavanones, and a chloroflavanone were used for the clonogenicity assay as racemates (Table I). They were purchased from Indofine Chemical Co., Inc. (Hillsborough, NJ).

Biological data. To examine the effects of flavanone derivatives on the clonogenicity of HCT116 human colorectal carcinoma cells, a long-term survival assay was performed. HCT116 cells were obtained from the American Type Culture Collection (ATCC) and maintained in DMEM (Invitrogen Life Technologies, Carlsbad, CA) supplemented with $10 \%$ heatinactivated fetal bovine serum (Invitrogen Life Technologies). Mid-log phase cultured HCT116 cells $\left(5 \times 10^{3}\right.$ cells/well) were seeded onto 24-well tissue culture plates (BD Falcon) in the absence or presence of different concentrations of flavanone derivatives and incubated for 7 days. Colonies formed were fixed with $6 \%$ glutaraldehyde, followed by staining with $0.1 \%$ crystal violet, as previously described (18). Following exposure of HCT116 cells to $20 \mu \mathrm{M}$ 5-hydroxy-7-methoxyflavanone (5-OH-7-OMeFlav) for $48 \mathrm{~h}$, cells were washed once with PBS and trypsinized. Cells were then incubated with $2.5 \mu \mathrm{g} / \mathrm{ml}$ propidium iodide (PI) for $5 \mathrm{~min}$ at room temperature. To quantify the number of dead cells, PI-stained cells were analyzed using a FACSCalibur flow cytometer (Becton Dickinson Immunocytometry Systems, San Jose, CA). HCT116 cells were treated with $20 \mathrm{mM}$ 5-OH-7-OMeFlav for 24 or $48 \mathrm{~h}$, fixed in $70 \%$ ethanol, washed twice with phosphatebuffered saline, and stained with $50 \mathrm{mg} / \mathrm{ml} \mathrm{PI}$, as previously described (19). The cellular DNA content was analyzed using a FACSCalibur flow cytometer. HCT116 cells were treated with either vehicle or $20 \mu \mathrm{M} 5-\mathrm{OH}-7 \mathrm{MeOFlav}$. At 24 or $28 \mathrm{~h}$ posttreatment, cells were collected and extracted in $20 \mathrm{mM}$ HEPES (pH 7.2) containing $1 \%$ Triton X-100, $10 \%$ glycerol, $150 \mathrm{mM}$ $\mathrm{NaCl}, 10 \mu \mathrm{g} / \mathrm{ml}$ leupeptin, and $1 \mathrm{mM}$ phenylmethylsulfonyl fluoride. The protein samples ( $20 \mu \mathrm{g} / \mathrm{lane})$ were then separated by $10 \%$ SDS-PAGE and transferred onto nitrocellulose filters. Western blotting was performed according to standard procedures using an antibody specific for p21 $(1: 1,000)$, cyclin D1 $(1: 2,000)$, PCNA $(1: 2,000)$, cyclin A $(1: 1,000)$, cyclin B $(1: 1,000)$ or GAPDH $(1: 2,000)$. All antibodies used in this study were obtained from Santa Cruz Biotechnology (Santa Cruz, CA). The signals were developed using an enhanced chemiluminescence detection system (Amersham Pharmacia Biotech Inc., Piscataway, NJ).

Three dimensional structure-activity relationships. To elucidate the SAR, 3 dimensional (3D) QSAR was conducted using the SYBYL 7.3 software (Tripos, St. Louis, MO). Twenty-six compounds were divided into 2 groups arbitrarily: a training set used to generate QSAR models, and a test set (compounds $8,15,16,18$, and 19 in Table I) used to validate the models. The training set was aligned using the SYBYL/Database Alignment module. In the alignment procedure, derivative 1, 2'-hydroxyflavanone, which showed the highest inhibitory activity against the clonogenicity of HCT116 cells, was used as a template and the atom-based root mean square fit method was adapted with the SYBYL/Database Align option (20).

\section{Results}

Clonogenic survival assay. The results of the clonogenic survival assay using HCT116 colorectal cancer cells treated with the flavanone derivatives are shown in Fig. 1 and their values obtained from densitometry analysis are listed in Table I. A low value obtained from densitometry analysis was indicative of better activity because the treated compound more effectively killed the HCT116 colorectal cancer cells. When the value of the non-treated cell was 500, the biological data ranged between 161.13 and 432.32 , which corresponded to an inhibitory effect of 67.8 and $13.5 \%$, respectively. The lowest inhibitory effect was caused by 2'-hydroxyflavanone and the strongest inhibitory effect was caused by 3,3',4',5,7-pentahydroxyflavanone (taxifolin).

Structure construction and optimization. Flavanone compounds have a C6-C3-C6 skeleton composed of 3 rings, A-, C-, and B-, respectively, and a chiral carbon at the C-3 position; thus, their 3D structure should be determined. (2S)-Naringenin contained in the crystallographic structure of chalcone isomerase (1eyq.pdb) was used as a template for the 3D structure calculations of the flavanone derivatives used in this experiment (21). The structures of 26 derivatives listed in Table I were built using SYBYL 7.3. All derivatives were used for their biological test as racemates, their $2 \mathrm{~S}$ conformations were used in silico experiments. Each structure was subjected to energy minimization, which was terminated upon the convergence of total energy $(0.05 \mathrm{kcal} / \mathrm{mol} \AA)$. Systematic conformational searches were performed using the SYBYL program to obtain the conformer with the lowest energy.

3D QSAR study. The 3D QSAR was carried out using comparative molecular field analyses (CoMFA) and comparative molecular similarity indices analysis (CoMSIA). Twenty-six flavanone derivatives were split-up into a training set to create the QSAR models and a test set to validate the models. Of the several CoMFA models, the model showing the best crossvalidated correlation coefficient $\left(\mathrm{q}^{2}=0.660\right)$ was chosen for further analyses. Partial least square analyses were performed to establish a linear relationship between the inhibition of clonogenicity and the resulting field matrix of the flavanone derivatives. The cross-validated analyses were performed using the leave-one-out (LOO) method. The final non-cross-validated $\left(\mathrm{r}^{2}=0.968\right)$ analysis was performed using the optimal number of components (6) obtained from the LOO method. In the partial least square analysis, the standard error of estimate and F-values were 15.459 and 69.732, respectively. The best CoMFA model was obtained using the region-focusing method. CoMFA models were evaluated by predicting the activity of each compound based on the model, and comparing the predictions 


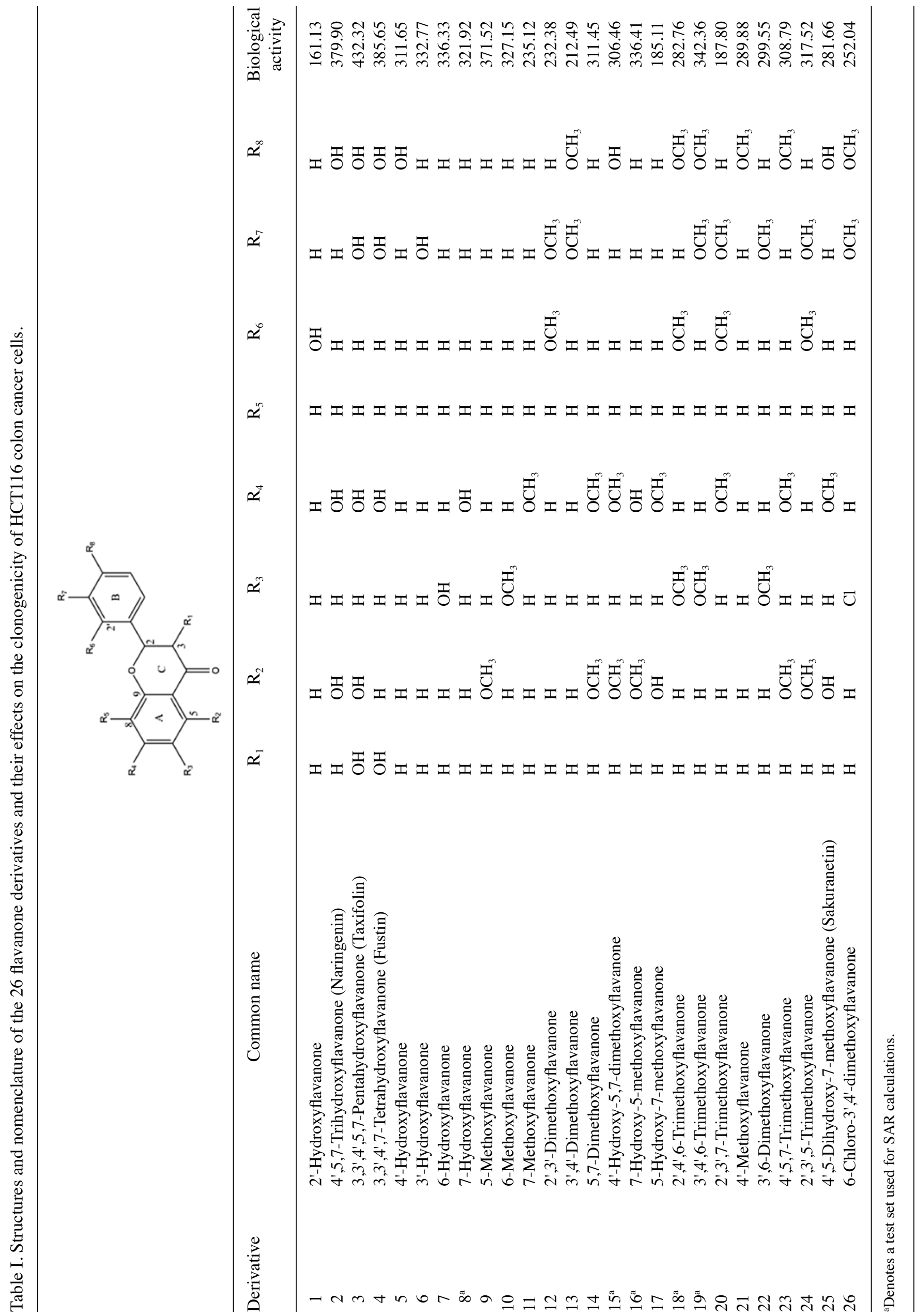


(A)

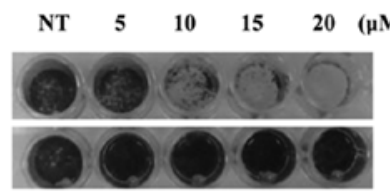

3,3',4',5,7-Pentahydroxyflavanone (3)

(B)

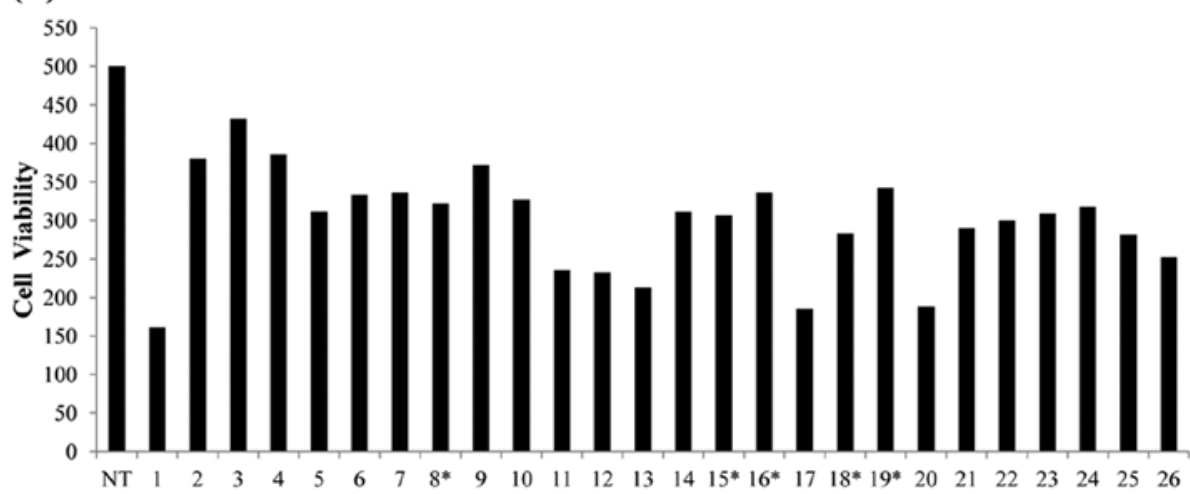

Figure 1. Effects of flavanone derivatives on the clonogenicity of HCT116 cells. HCT116 cells $\left(5 \times 10^{3}\right.$ cells/well) were plated and cultured for 7 days in the absence or presence of different concentrations of flavanone derivatives. Similar results were obtained from 2 other independent experiments. (A) The greatest inhibitory effect was observed for derivative 1 and the lowest effect was observed for derivative 3, and (B) values obtained from the densitometry analysis. ${ }^{*}$ Test set used for the SAR calculations.

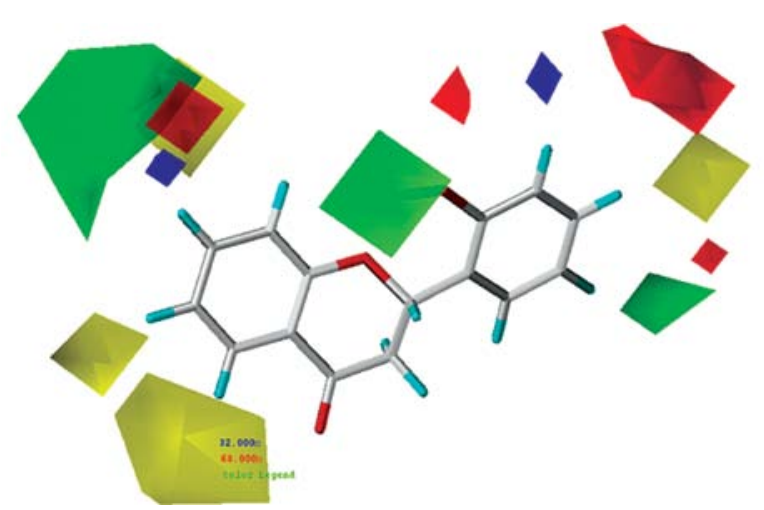

Figure 2. CoMFA contour maps. The corresponding steric and electrostatic field contributions were 57.4 and $42.6 \%$, respectively. The steric field contours are shown in green (more bulk-favored) and yellow (less bulk-favored), while the electrostatic field contours are shown in red (electronegative substituentfavored, 68\%) and blue (electropositive substituent-favored, 32\%).

with the experimental data. Residuals between the experimental and predicted values for the training set ranged from $0.07 \%$ (derivative 10,6-methoxyflavanone) to $14.2 \%$ (derivative 20, 2',3'7-trimethoxyflavanone) and this error range was acceptable. To validate the QSAR model, 5 derivatives, 8, 15, 16, 18, and 19, were selected as a test set. Their residuals ranged between $0.1 \%$ (derivative 8,7-hydroxyflavanone) and $18.5 \%$ (derivative 19, 3',4',6-trimethoxyflavanone); thus, this QSAR model was reliable.

To visualize the relationship between the structures of the flavanone derivatives and the inhibition of clonogenicity, CoMFA contour maps were generated using SYBYL 7.3. The steric and electrostatic field descriptors contributed 57.4 and $42.6 \%$, respectively. Of the steric field contributors, the bulky favored and disfavored regions contributed 35 and $65 \%$, respectively (Fig. 2). Of the electrostatic field contributors, the

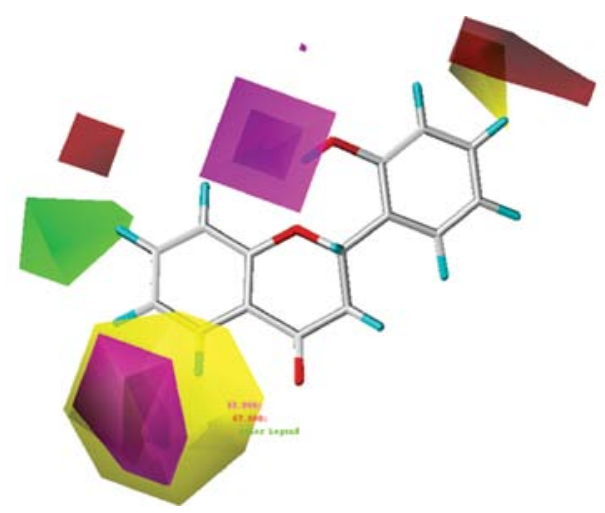

Figure 3. The field contributions of steric and $\mathrm{H}$-bond acceptor fields were 54.7 and $45.3 \%$, respectively in the CoMSIA contour maps. The more bulk favored region and less bulk favored regions are shown in green and yellow, respectively. The $\mathrm{H}$-bond acceptor favored contour and disfavored contour are colored in magenta (33\%) and red (67\%), respectively.

electronegative and electropositive favored regions contributed 32 and $68 \%$, respectively.

While CoMFA provides information on only the steric and electrostatic effects, CoMSIA can yield information on the steric and electrostatic effects as well as the hydrogen bond effects; thus, CoMSIA analysis was performed. A partial least squares analysis was used to determine correlations between the biological activity and descriptors of the physicochemical property of each derivative. The model displaying the best cross-validated value $\left(\mathrm{q}^{2}=0.556\right)$ was selected. The corresponding $\mathrm{r}^{2}$ was 0.945 . The number of components, the standard error of estimate, and the F-value were 4, 18.851, and 68.702, respectively. A comparison of the experimental data and of the values predicted by CoMSIA was performed. In the training set, the residual errors between the experimental values and the predicted values ranged between $0.2 \%$ (derivative 3 , 
A
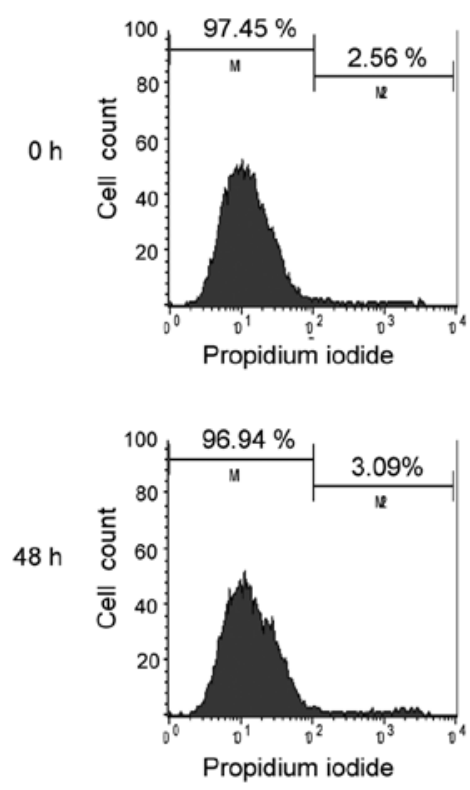

B

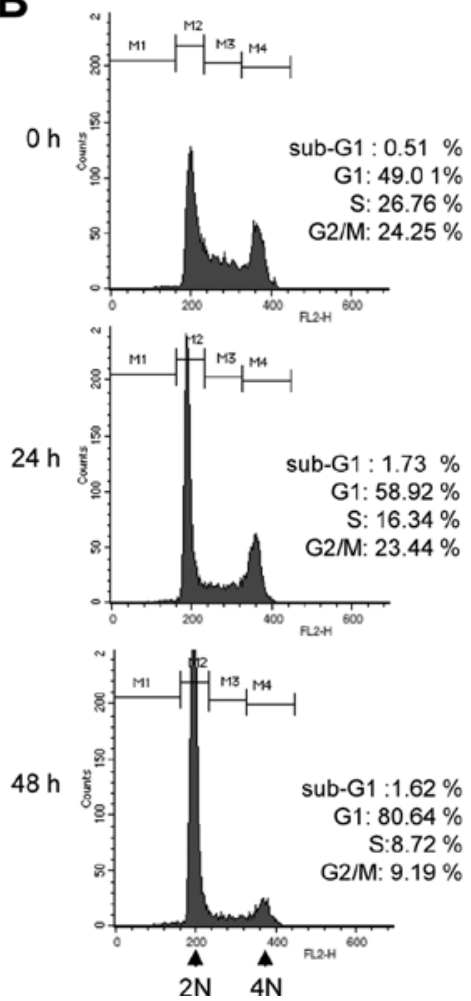

C

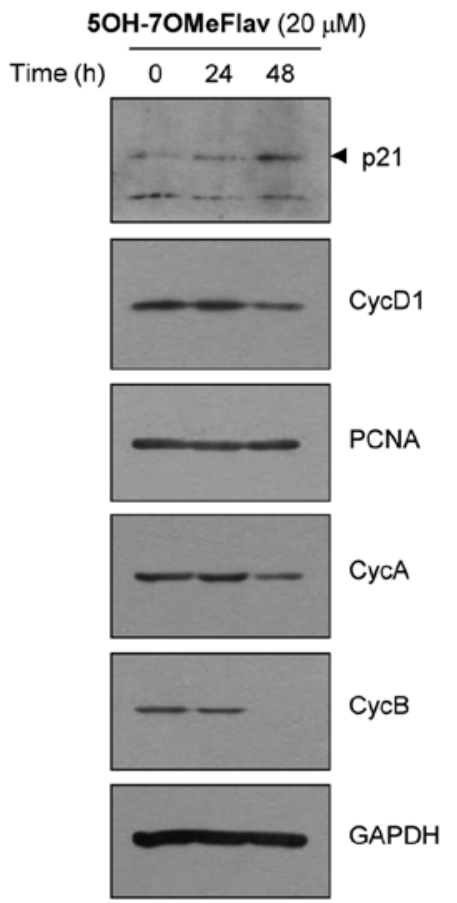

Figure 4. Effects of the 5-OH-7-OMeFlav on the inhibition of G1 cell cycle progression. (A) HCT116 cells were treated with 20 $\mu \mathrm{M}$ 5-OH-7-OMeFlav for $48 \mathrm{~h}$, and the population of PI-positive cells was quantified using FACSCalibur flow cytometry. M1, PI-negative live cells; M2, PI-positive dead cells. (B) HCT116 cells were treated with $20 \mu \mathrm{M} 5-\mathrm{OH}-7-\mathrm{OMeFlav}$ for 24 or $48 \mathrm{~h}$. DNA content was analyzed by PI staining at $488 \mathrm{~nm}$ using FACSCalibur flow cytometry. M1, sub-G1; M2, G1; M3, S; M4, G2/M; 2N, diploid; 4N, tetraploid. (C) HCT116 cells were treated with $20 \mu \mathrm{M}$ 5-OH-7-OMeFlav for 24 or $48 \mathrm{~h}$, and the expression of cell cycle regulatory proteins in whole cell lysates were assessed by Western blotting using the indicated antibodies. GAPDH was used as an internal control.

3,3',4',5,7-pentahydroxyflavanone) and $20.0 \%$ (derivative 17 , 5-hydroxy-7methoxyflavanone), and in the case of the test set, they were $4.2 \%$ (derivative 18,2 ', 4',6-trimethoxyflavanone) and $18.4 \%$ (derivative 19, 3',4',6-trimethoxyflavanone). These results demonstrate that the CoMSIA model used in this experiment was reliable.

The CoMSIA calculations revealed that the steric and H-bond acceptor fields contribute 54.7 and 45.3\%, respectively. Like CoMFA, the CoMSIA contour map was generated and is shown in Fig. 3. In the steric fields, the bulky favored region and the disfavored region contributed 33 and $67 \%$, respectively. Similarly to the steric fields, the H-bond acceptorfavored region and disfavored region contributed 33 and $67 \%$, respectively.

The substituent at C-5 favors the non-bulky group and the functional group as the H-bond acceptor. The activity of derivative 17 was better than that of derivative 14. Likewise, derivative 25 displayed better activity than derivative 15 . In the cases of derivatives 26 and 19, the former showed better activity than the later because C- 6 favors the non-bulky group. The C-7 position favors a bulky substituent such as methoxyl group. For example, derivative 11 showed better activity than derivative 8 . Derivatives 1,12 , and 20 produced good inhibitory effects because the C-2' prefers a hydroxyl or methoxyl group. When 2 bulky groups are attached to the B-ring and are separated they do not show good activity. The ortho substituted dimethoxyl groups (derivatives 12, 13, 20, and 26) showed good activity.

\section{Discussion}

To investigate the molecular mechanism underlying the inhibition of clonogenicity of HCT116 cells, the flavanone derivatives showing good inhibitory activity, derivative 17 (5-hydroxy-7-methoxyflavanone, 5-OH-7-OMeFlav), was selected, and its effect on the induction of cell death was evaluated. PI is a fluorescent dye that can bind to nucleotides by intercalating between the bases. PI is impermeable and excluded from viable cells; however, in cells lacking membrane integrity, fluorescent PI rapidly diffuses out of the cell (22). Thus, PI staining is widely used to identify dead cells in a solution. Flow cytometry analysis showed that the population of PI-stained cells only slightly increased following treatment with $20 \mu \mathrm{M}$ 5-OH-7-OMeFlav for $48 \mathrm{~h}$ (Fig. 4A), suggesting that the flavanone derivatives had no effect on cell death. We then examined the effect of the 5-OH-7-OMeFlav on the progression of the cell cycle using FACS analysis. The number of sub-G1 cells was not changed by $5-\mathrm{OH}-7-\mathrm{OMeFlav}$ treatment (Fig. 4B), further conforming that this compound had no effect on inducing apoptotic cell death. Forty-eight hours after 5-OH-7-OMeFlav treatment, the number of cells increased in the G1 phase (49.0\% vs. $80.64 \%)$ with concomitant decreases in the $\mathrm{S}$ phase $(26.76 \%$ vs. $8.72 \%)$ and $\mathrm{G} 2 / \mathrm{M}$ phase $(24.25 \%$ vs. $9.19 \%$ ) of the cell cycle when compared to the control cells (Fig. 4B). These data suggest that 5-OH-7-OMeFlav treatment arrested the cell cycle progression at the G1 phase for HCT116 cells. We next analyzed the effect of the 5-OH-7-OMeFlav on 
the expression of cell cycle regulators by Western blot analysis. In this analysis, the steady-state level of $\mathrm{p} 21^{\text {Wafl//ip1 }}$ (hereafter p21) was increased, whereas the levels of cyclin D1, cyclin A, and cyclin $\mathrm{B} 1$ proteins were decreased when compared to the control cells (Fig. 4C). PCNA and GAPDH were not affected. Since p21 plays important roles in the inhibition of cell cycle progression at $\mathrm{G} 1$ and $\mathrm{G} 2 / \mathrm{M}$ phases $(23,24)$ and cyclin D1 regulates the transition of cells from the G1 to $\mathrm{S}$ phase (25), it seems likely that the 5-OH-7MeOFlav-induced cell cycle arrest at the G1 phase in HCT116 cells was mediated by the up-regulation of p21 and down-regulation of cyclin D1. Taken together, our results suggest that flavanone derivatives control the expression of cell cycle regulatory proteins, which blocks G1 cell cycle progression and inhibits the clonogenicity of HCT116 cells. As mentioned above, the results obtained from this QSAR study reveal the structural condition to show good inhibitory effects on HCT116 colorectal cancer cells, so that compounds that have met the condition will be designed, prepared, and assessed in a future study.

\section{Acknowledgements}

This study was supported by the Priority Research Centers Program (NRF, 2009-0093824), Agenda program (RDA, 8-2152), the Bio \& Medical Technology Development Program (NRF, 2011-0027754), the next generation Biogreen21 program (RDA, PJ007982), and the NRF funded by MEST (2010-0020966).

\section{References}

1. Goldwasser J, Cohen PY, Lin W, Kitsberg D, Balaguer P, Polyak SJ, Chung RT, Yarmush ML and Nahmias Y: Naringenin inhibits the assembly and long-term production of infectious hepatitis $\mathrm{C}$ virus particles through a PPAR-mediated mechanism. J Hepatol 55 963-971, 2011.

2. Xu YJ, Capistrano R I, Dhooghe L, Foubert K, Lemière F, Maregesi S, Baldé A, Apers S and Pieters L: Herbal medicines and infectious diseases: characterization by LC-SPE-NMR of some medicinal plant extracts used against malaria. Planta Med 77: 1139-1148, 2011.

3. Choi SE, Park KH, Han BH, Jeong MS, Seo SJ, Lee DI, Joo SS and Lee MW: Inhibition of inducible nitric oxide synthase and cyclooxygenase- 2 expression by phenolic compounds from roots of Rhododendron mucronulatum. Phytother Res 25: 1301-1305, 2011.

4. Sheu MJ, Chou PY, Cheng HC, Wu CH, Huang GJ, Wang BS, Chen JS, Chien YC and Huang MH: Analgesic and antiinflammatory activities of a water extract of Trachelospermum jasminoides (Apocynaceae). J Ethnopharmacol 126: 332-338, 2009.

5. Jin CH, Shin EJ, Park JB, Jang CG, Li Z, Kim MS, Koo KH, Yoon HJ, Park SJ, Choi WC, et al: Fustin flavonoid attenuates $\beta$-amyloid (1-42)-induced learning impairment. J Neurosci Res 87: 3658-3670, 2009

6. Park BC, Lee YS, Park HJ, Kwak MK, Yoo BK, Kim JY and Kim JA: Protective effects of fustin, a flavonoid from Rhus verniciflua stokes, on 6-hydroxydopamine-induced neuronal cel death. Exp Mol Med 39: 316-326, 2007.

7. Park KY, Jung GO, Lee KT, Choi J, Choi MY, Kim GT, Jung HJ and Park HJ: Antimutagenic activity of flavonoids from the heartwood of Rhus verniciflua. J Ethnopharmacol 90: 73-79, 2004.
8. Shi LL, Chen BN, Gao M, Zhang HA, Li YJ, Wang L and Du GH: The characteristics of therapeutic effect of pinocembrin in transient global brain ischemia/reperfusion rats. Life Sci 88: 521-528, 2011.

9. Punvittayagul C, Wongpoomchai R, Taya S and Pompimon W: Effect of pinocembrin isolated from Boesenbergia pandurata on xenobiotic-metabolizing enzymes in rat liver. Drug Metab Lett 5: 1-5, 2011.

10. Diaz Napal GN, Defagó MT, Valladares GR and Palacios SM: Response of Epilachna paenulata to two flavonoids, pinocembrin and quercetin, in a comparative study. J Chem Ecol 36: 898-904, 2010.

11. Malek SN, Phang CW, Ibrahim H, Norhanom AW and Sim KS: Phytochemical and cytotoxic investigations of Alpinia mutica rhizomes. Molecules 16: 583-589, 2011.

12. Wan L, Sun X, Wang X, Li Y, Yu Q and Guo C: A stereospecific HPLC method and its application in determination of pharmacokinetics profile of two enantiomers of naringenin in rats. J Chromatogr Sci 49: 316-320, 2011.

13. Vega-Villa KR, Remsberg CM, Takemoto JK, Ohgami Y, Yáñez JA, Andrews PK and Davies NM: Stereospecific pharmacokinetics of racemic homoeriodictyol, isosakuranetin, and taxifolin in rats and their disposition in fruit. Chirality 23: 339-348, 2011.

14. Santos FC, Abreu P, Castro HC, Paixão IC, Cirne-Santos CC, Giongo V, Barbosa JE, Simonetti BR, Garrido V, Bou-Habib DC, et al: Synthesis, antiviral activity and molecular modeling of oxoquinoline derivatives. Bioorg Med Chem 17: 5476-5481, 2009.

15. Meyerhardt JA: Beyond standard adjuvant therapy for colon cancer: role of nonstandard interventions. Semin Oncol 38: 533-541, 2011.

16. de Gramont A, de Gramont A, Chibaudel B, Bachet JB, Larsen AK, Tournigand C, Louvet $\mathrm{C}$ and André T: GERCOR from chemotherapy to targeted therapy in adjuvant treatment for stage III colon cancer. Semin Oncol 38: 521-532, 2011.

17. Komatsu Y, Yuki S, Sogabe S, Fukushima H, Iwanaga I, Kudo M, Tateyama M, Meguro T, Uebayashi M, Saga A, Sakata Y and Asaka M: Phase II study of combined treatment with irinotecan and S-1 (IRIS) in patients with inoperable or recurrent advanced colorectal cancer (HGCSG0302). Oncology 80: 70-75, 2011.

18. Franken NA, Rodermond HM, Stap J, Haveman J and van Bree C: Clonogenic assay of cells in vitro. Nat Protoc 1: 2315-2319, 2006 .

19. Shin SY, Kim CG, Kim SH, Kim YS, Lim Y and Lee YH: Chlorpromazine activates $\mathrm{p} 21^{\text {Waf } / / \text { Cipl }}$ gene transcription via early growth response-1 (Egr-1) in C6 glioma cells. Exp Mol Med 42: 395-405, 2010.

20. Lee S, Woo Y, Shin SY, Lee YH and Lim Y: Relationships between the structures of flavanone derivatives and their effects in enhancing early growth response-1 gene expression. Bioorg Med Chem Lett 19: 2116-2120, 2009.

21. Jez JM, Bowman ME, Dixon RA and Noel JP: Structure and mechanism of the evolutionarily unique plant enzyme chalcone isomerase. Nat Struct Biol 7: 786-791, 2000.

22. Ross DD, Joneckis CC, Ordóñez JV, Sisk AM, Wu RK, Hamburger AW Nora RE and Nora RE: Estimation of cell survival by flow cytometric quantification of fluorescein diacetate/ propidium iodide viable cell number. Cancer Res 49: 3776-3782, 1989.

23. Harper JW, Adami GR, Wei N, Keyomarsi K and Elledge SJ: The p21 cdk-interacting protein cipl is a potent inhibitor of Gl cyclin-dependent kinases. Cell 75: 805-816, 1993.

24. Bunz F, Dutriaux A, Lengauer C, Waldman T, Zhou S, Brown JP, Sedivy JM, Kinzler KW and Vogelstein B: Requirement for p53 and p21 to sustain G2 arrest after DNA damage. Science 282: 1497-1501, 1998.

25. Hinds PW, Dowdy SF, Eaton EN, Arnold A and Weinberg RA: Function of a human cyclin gene as an oncogene. Proc Natl Acad Sci USA 91: 709-713, 1994. 